\title{
'Surprise': Outbreak of Campylobacter infection associated with chicken liver pâté at a surprise birthday party, Adelaide, Australia, 2012
}

\author{
Amy Parry, ab Emily Fearnley ab and Emma Denehy ${ }^{a}$ \\ Correspondences to Amy Parry (e-mail: amy.parry@health.sa.gov.au)
}

Objective: In July 2012, an outbreak of Campylobacter infection was investigated by the South Australian Communicable Disease Control Branch and Food Policy and Programs Branch. The initial notification identified illness at a surprise birthday party held at a restaurant on 14 July 2012. The objective of the investigation was to identify the potential source of infection and institute appropriate intervention strategies to prevent further illness.

Methods: A guest list was obtained and a retrospective cohort study undertaken. A combination of paper-based and telephone questionnaires were used to collect exposure and outcome information. An environmental investigation was conducted by Food Policy and Programs Branch at the implicated premises.

Results: All 57 guests completed the questionnaire (100\% response rate), and 15 met the case definition. Analysis showed a significant association between illness and consumption of chicken liver pâté (relative risk: 16.7, 95\% confidence interval: 2.4-118.6). No other food or beverage served at the party was associated with illness. Three guests submitted stool samples; all were positive for Campylobacter. The environmental investigation identified that the cooking process used in the preparation of chicken liver pâté may have been inconsistent, resulting in some portions not cooked adequately to inactivate potential Campylobacter contamination.

Discussion: Chicken liver products are a known source of Campylobacter infection; therefore, education of food handlers remains a high priority. To better identify outbreaks among the large number of Campylobacter notifications, routine typing of Campylobacter isolates is recommended.

\section{C} ampylobacteriosis is the most commonly reported notifiable infectious gastrointestinal disease in Australia, with annual national notification rates of between 104.8 and 117.3 per 100000 during the period 2007-2011 (excluding New South Wales, the largest state in Australia). ${ }^{1}$ Notification rates are similar in South Australia and other developed countries. ${ }^{2,3}$ True case numbers are most likely higher due to an estimated $90 \%$ of campylobacteriosis cases not being reported. ${ }^{4}$ Despite high case numbers, campylobacteriosis outbreaks are uncommon. ${ }^{2,3,5-7}$ One key reason for this is the limited microbiological speciation and typing of specimens.

Campylobacter is a bacterium commonly found in raw poultry ${ }^{8,9}$ and other sources, including contaminated water, unpasteurized milk and pets. ${ }^{10}$ The incubation period of Campylobacter infection is usually between two and five days, but can range from one to 10 days. ${ }^{11}$ Symptoms include diarrhoea, fever and abdominal pains. ${ }^{11}$ The infective dose is reportedly low, with 500 organisms required to cause illness. ${ }^{5,7}$

\section{On 27 July 2012, the South Australian} Communicable Disease Control Branch was notified of a suspected campylobacteriosis outbreak linked to a surprise birthday party at a restaurant on 14 July 2012. The index case reported that other guests were also ill after the party. A retrospective cohort study was conducted among the 57 guests. The investigation objectives were to define the outbreak by person, time and place; identify the potential source of infection; and institute intervention strategies to prevent further illness.

\footnotetext{
Communicable Disease Control Branch, South Australian Department of Health and Ageing, Adelaide, South Australia, Australia.

OzFoodNet, South Australian Department of Health and Ageing, Adelaide, South Australia, Australia.

Submitted: 12 October 2012; Published: 13 November 2012

doi: 10.5365/wpsar.2012.3.4.011
} 
Figure 1. Reported illness by date of onset after attending a surprise birthday party, South Australia, July 2012 $(n=15)$

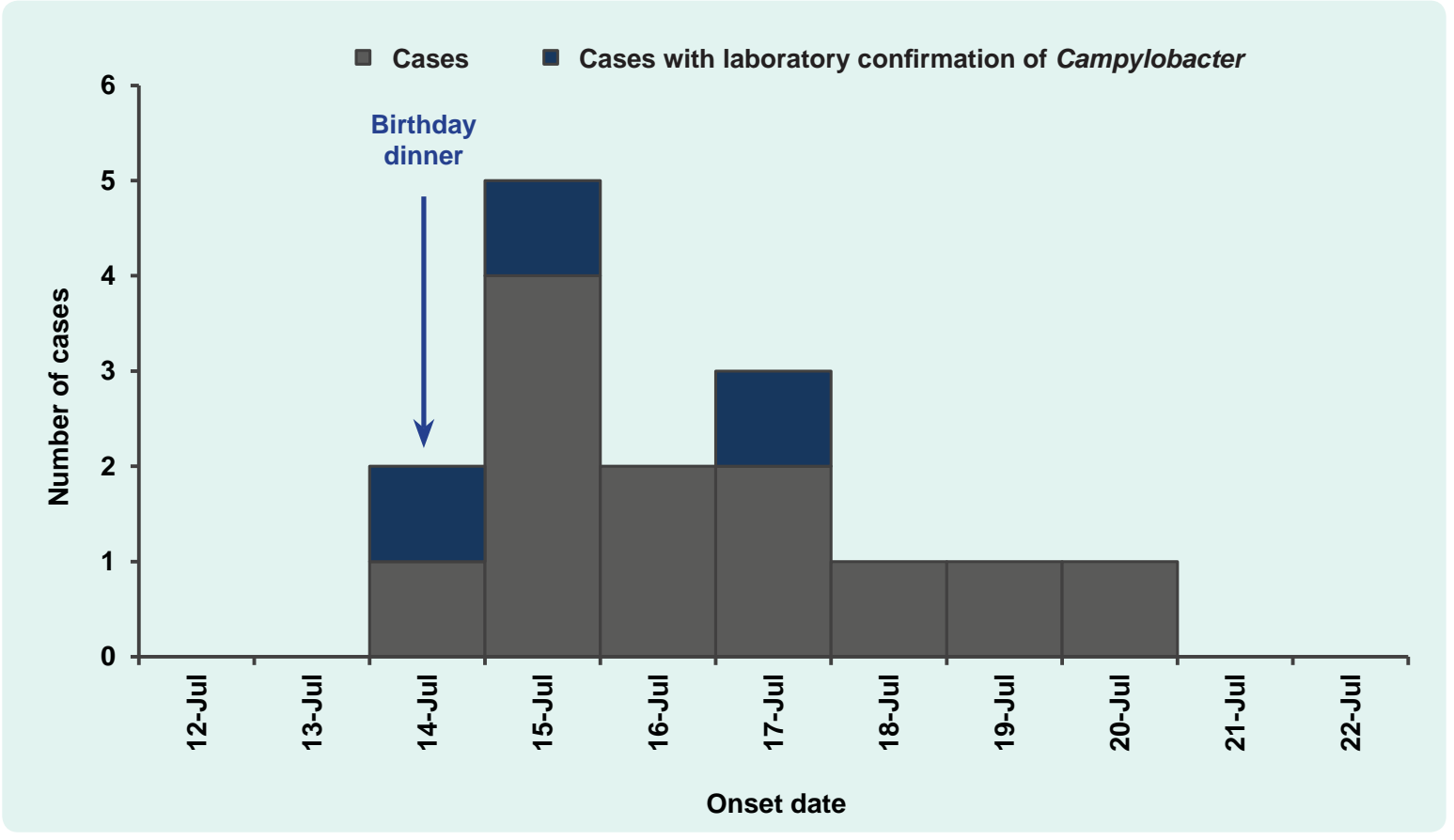

\section{METHODS}

A retrospective cohort study was conducted among the 57 guests at the surprise birthday party at a restaurant on 14 July 2012. The questionnaire collected demographic information, personal and household gastrointestinal illness before and after the event, food and beverage consumption at the party and other common events attended. Presentation to a medical practitioner and hospitalization information were also obtained. Guest contact details (a combination of telephone numbers and postal addresses) were provided by the event organizer. The questionnaire was completed by trained interviewers via telephone or through self-completion of paper-based questionnaires, with all interviews completed within 14 days. Guests contacted via post were invited to telephone the investigation team to complete their questionnaire.

A case was defined as any person who consumed food and/or beverages at the birthday party on 14 July 2012 and subsequently reported diarrhoea (three or more loose stools in a 24 -hour period).

Data analysis was conducted with STATA 12. Univariate analysis included attack rates, $p$-values, relative risks and 95\% confidence intervals (exact). A $p$-value of less than 0.05 was considered significant. chi-squared (exact) tests were used to test for significance between categorical variables. Additional analysis was conducted excluding the cases that reported illness within 24 hours to account for the possibility that their illness was not associated with this outbreak. As this did not affect the outcome, this analysis was excluded.

The Food Policy and Programs Branch conducted an environmental investigation at the implicated premises. As there was no left-over product, information on ingredients and cooking processes of foods served were obtained.

\section{RESULTS}

All 57 guests responded to the investigation questionnaire (100\% response rate). Fifteen met the case definition. The epidemic curve (Figure 1) indicates illness onset was rapid, with two cases reporting illness later on the night of the party and five reporting illness early the next morning. Eight of the 15 cases sought medical care, three of whom provided faecal specimens. All three faecal specimens were confirmed via culture with Campylobacter infection; one was further speciated as Campylobacter jejuni. One case reported hospitalization.

After diarrhoea, the most commonly described symptom for all 15 cases was abdominal pains (85.7\%). 
Table 1. Univariate food frequency analysis from surprise birthday party, South Australia, July $2012(n=57)$

\begin{tabular}{|c|c|c|c|c|c|c|c|c|c|c|}
\hline \multirow{2}{*}{ Meal } & \multirow{2}{*}{ Exposure } & \multicolumn{3}{|c|}{ Exposed } & \multicolumn{3}{|c|}{ Unexposed } & \multirow[b]{2}{*}{$\mathbf{R R}$} & \multirow[b]{2}{*}{$95 \% \mathrm{Cl}$} & \multirow[b]{2}{*}{$p$-value } \\
\hline & & No. ill & Total & AR\% & No. ill & Total & AR\% & & & \\
\hline \multirow[t]{2}{*}{ Starter } & Nuts & 2 & 13 & 15.4 & 12 & 39 & 30.8 & 0.5 & $0.1-2.0$ & 0.279 \\
\hline & Olives & 3 & 12 & 33.3 & 11 & 43 & 25.6 & 1.3 & $0.5-3.34$ & 0.594 \\
\hline \multirow[t]{2}{*}{ Entrée } & Chicken liver pâté & 14 & 26 & 53.8 & 1 & 31 & 3.2 & 16.7 & $2.4-118.6$ & $<0.001$ \\
\hline & Mussels marinière & 1 & 29 & 3.5 & 14 & 28 & 50.0 & 0.1 & $0.0-0.5$ & $<0.001$ \\
\hline \multirow[t]{2}{*}{ Main course } & $\begin{array}{l}\text { Duck fillet with orange } \\
\text { glaze }\end{array}$ & 6 & 24 & 25.0 & 9 & 33 & 27.3 & 0.9 & $0.4-2.2$ & 0.780 \\
\hline & $\begin{array}{l}\text { Chateaubriand with } \\
\text { béarnaise sauce }\end{array}$ & 9 & 33 & 27.3 & 6 & 23 & 26.1 & 1.1 & $0.4-2.5$ & 0.921 \\
\hline \multirow[t]{2}{*}{ Dessert } & $\begin{array}{l}\text { Flourless chocolate } \\
\text { cake }\end{array}$ & 6 & 23 & 26.1 & 9 & 34 & 26.5 & 1.0 & $0.4-2.4$ & 0.974 \\
\hline & Rhubarb crème brûlée & 11 & 35 & 31.4 & 4 & 22 & 18.2 & 1.7 & $0.6-4.8$ & 0.269 \\
\hline \multirow[t]{2}{*}{ After dinner } & $\begin{array}{l}\text { Chocolate sponge } \\
\text { birthday cake }\end{array}$ & 1 & 13 & 7.7 & 14 & 43 & 32.6 & 0.2 & $0.0-1.6$ & 0.076 \\
\hline & Chocolates & 3 & 9 & 33.3 & 10 & 35 & 28. 6 & 1.2 & $0.4-3.4$ & 0.780 \\
\hline
\end{tabular}

$\mathrm{AR}$ - attack rate; $\mathrm{RR}$ - relative risk; $\mathrm{Cl}$ - confidence interval.

No vomiting was reported. The median duration of illness for those recovered at the time of interview $(n=12)$ was eight days.

Two children under 18 years attended the party; remaining guests were adults $(40 \%$ aged between 40 and 49 years). The sex distribution of people attending the party was even (females $51 \%$ ). There was no statistical difference between illness and sex or age.

The menu served at the party consisted of two options each for entrée, main course and dessert. Consumption of the chicken liver pâté entrée was significantly associated with illness (relative risk [RR]: 16.7, 95\% confidence interval [CI]: 2.4-118.6), whereas consumption of mussels for entrée was inversely associated with illness (RR: $0.1,95 \% \mathrm{Cl}: 0.0-0.5$ ). No other foods or beverages served at the party were associated with illness (Table 1 ). Nine males reported eating pâté compared to 17 females, of which five males and 10 females were cases (1:2).

The environmental investigation team identified that livers used in the pâté were sautéed during preparation; actual duration of cooking was not recorded.

\section{DISCUSSION}

Consumption of chicken liver pâté was associated with the illness at the surprise birthday party. The reduced risk of illness associated with consumption of the mussel marinière was likely due to the dichotomous nature of the menu choices rather than a true biological association.

Studies have reported that livers can be both internally and externally contaminated with Campylobacter. ${ }^{8,9}$ The presence of Campylobacter in chicken livers can be reduced by cooking them for two to three minutes after they reach a core temperature of $70^{\circ} \mathrm{C}$ to $80^{\circ} \mathrm{C}$. $^{8}$ The restaurant reported only sautéing the livers before making the pâté and that a large volume of pâté was made and stored in batches. It is plausible that multiple batches of pâté were served that evening, some contaminated and others not. Insufficient cooking of the chicken livers was most likely a significant causative factor in this outbreak.

Cases reported rapid onset of illness after the event, shorter than the usual incubation period for campylobacteriosis, perhaps due to a large concentration of bacteria in the pâté ${ }^{12}$ or an increased virulence of the strain. Susceptibility to Campylobacter infection is influenced by host immunosuppression, stomach acidity and antibiotic and proton pump inhibitor use..$^{7,12,13}$

Pâté as the vehicle for Campylobacter infection is not a new finding. Numerous Campylobacter outbreaks throughout Australia ${ }^{9}$ and internationally ${ }^{2,3,5}$ have also identified liver pâté as the possible vehicle. Outbreaks of this kind may be greatly underestimated due to inherent difficulties in detecting Campylobacter outbreaks in Australia. ${ }^{6}$ Campylobacter isolates are not routinely subtyped despite molecular subtyping methods being 
available to identify reasonable genetic diversity in human cases. ${ }^{14}$ Without subtyping, the identification of clusters and outbreaks is largely restricted to temporal or geographical associations or reliance on medical notifications identifying common events. ${ }^{15}$

As all guests responded to the questionnaire, there was no potential for selection bias. The majority of interviews were conducted in the first five days of the investigation, reducing recall bias. A potential investigation limitation of the study design was the use of a mixed method approach of telephone and paperbased questionnaires.

In summary, Campylobacter infection linked to liver pâté has been identified numerous times despite the cooking procedures necessary to ensure safe preparation of liver pâté being clearly described. The current high standards of hygiene and food preparation in Australia have minimized the impact of foodborne illness linked to commercial settings. This outbreak has demonstrated the importance of continuing education of Campylobacter infection, and the appropriate handling and cooking of higher risk foods, including livers. Furthermore, to better identify outbreaks among the large number of Campylobacter notifications, routine typing of Campylobacter isolates is recommended.

\section{Conflicts of interest}

None declared.

Funding

None.

\section{Acknowledgements}

The OzFoodNet programme is an initiative of the Australian Government Department of Health and Ageing. The authors would like to acknowledge the work of the investigation team and other members of the Department who provided advice. Thank you to the South Australia Health Disease Surveillance and Investigation Section: Annika Wendland, Helen Livissianos, Remy Boyes, Hannah Vogt and Pip Rokkas; the Food Standards Surveillance Section of Food Policy and Programs Branch: Glen Martin, Garry Clarke, Jamie Woodward, Vanessa McEvoy and
Alessia Centofanti; as well as Communicable Disease Control Branch doctors: Ann Koehler, Albert Lessing, Kushani Marshall and Jane Raupach.

\section{References:}

1. National Notifiable Disease Surveillance System. Canberra, Australian Government Department of Health and Ageing, 2012 (http://www9.health.gov.au/cda/Source/CDA-index.cfm, accessed 19 September 2012).

2. Little $\mathrm{CL}$ et al. A recipe for disaster: outbreaks of campylobacteriosis associated with poultry liver pâté in England and Wales. Epidemiology and Infection, 2010, 138:1691-1694. doi:10.1017/S0950268810001974 pmid:20727250

3. O'Leary MC et al. A continuous common-source outbreak of campylobacteriosis associated with changes to the preparation of chicken liver pâté. Epidemiology and Infection, 2009, 137:383-388. doi:10.1017/S0950268808001003 pmid: 18647437

4. Hall G et al. Estimating community incidence of Salmonella, Campylobacter, and Shiga toxin-producing Escherichia coli infections, Australia. Emerging Infectious Diseases, 2008, 14:1601-1609. doi:10.3201/eid1410.071042 pmid: 18826825

5. Inns T, Foster K, and Gorton R. Cohort study of a campylobacteriosis outbreak associated with chicken liver parfait, United Kingdom, June 2010. Euro surveillance: European Communicable Disease Bulletin, 2010, 15(44):pii=19704. pmid:21087588

6. Unicomb LE et al. Outbreaks of campylobacteriosis in Australia, 2001 to 2006. Foodborne Pathogens and Disease, 2009, 6:12411250. doi:10.1089/fpd.2009.0300 pmid:19895264

7. Janssen $\mathrm{R}$ et al. Host-pathogen interactions in Campylobacter infections: the host perspective. Clinical Microbiology Reviews, 2008, 21:505-518. doi:10.1128/CMR.00055-07 pmid: 18625685

8. Whyte R, Hudson JA, Graham C. Campylobacter in chicken livers and their destruction by pan frying. Letters in Applied Microbiology, 2006, 43:591-595. doi:10.1111/j.1472765X.2006.02020.x pmid: 17083702

9. Merritt T, Combs B, Pingault N. Campylobacter outbreaks associated with poultry liver dishes. Communicable Diseases Intelligence, 2011, 35:299-300. pmid:22624491

10. Tenkate TD, Stafford RJ. Risk factors for Campylobacter infection in infants and young children: a matched case-control study. Epidemiology and Infection, 2001, 127:399-404. doi:10.1017/ S0950268801006306 pmid:11811871

11. Heymann MD. Control of Communicable Diseases Manual $19^{\text {th }}$ Edition. Washington, World Health Organization and American Public Health Association, 2008.

12. Allos BM. Microbiology, pathogenesis, and epidemiology of Campylobacter infection, 9 August 2011 (http://www.uptodate. com/contents/microbiology-pathogenesis-and-epidemiology-ofcampylobacter-infection, accessed 24 September 2012).

13. Neal KR et al. Omeprazole as a risk factor for campylobacter gastroenteritis: case-control study. BMJ (Clinical research ed.), 1996, 312:414-415. doi:10.1136/bmj.312.7028.414 pmid:8601113

14. Mickan L et al.; Australian Campylobacter Subtyping Study Group. Multilocus sequence typing of Campylobacter jejuni isolates from New South Wales, Australia. Journal of Applied Microbiology, 2007, 102:144-152. doi:10.1111/j.1365-2672.2006.03049.x pmid: 17184329

15. Swaminathan B et al.; CDC PulseNet Task Force. PulseNet: the molecular subtyping network for foodborne bacterial disease surveillance, United States. Emerging Infectious Diseases, 2001, 7:382-389. pmid:11384513 